\title{
Casimir Force between Nanoparticles Immersed in a Crosslinked Polymer Blend
}

\author{
S. El Fassi, M. Benhamou*, M. Boughou, H. Kaidi, M. El Yaznasni \\ AND H. Ridounane \\ Laboratoire de Physique des Polymères et Phénomènes Critiques \\ Faculté des Sciences Ben M'sik, P.O. Box 7955, Casablanca, Morocco
}

(Received January 30, 2010; in final form July 22, 2010)

\begin{abstract}
We consider a crosslinked polymer blend made of two polymers of different chemical nature. We suppose that such a system incorporates small colloidal particles, which prefer to be attracted by one polymer, close to the spinodal temperature. This is the so-called critical adsorption. As assumption, the particle diameter, $d_{0}$, is considered to be small enough in comparison with the size of microdomains (mesh size) $\xi^{*} \sim a n^{1 / 2}$, with $a-$ the monomer size and $n$ - the number of monomers between consecutive crosslinks. The critical fluctuations of the crosslinked polymer mixture induce a pair-potential between particles located in the non-preferred phase. The purpose is the determination of the Casimir pair-potential, $U_{2}(r)$, as a function of the interparticle distance $r$. To achieve calculations, use is made of an extended de Gennes field theory that takes into account the colloid-polymer interactions. Within the framework of this theory, we first show that the pair-particle is attractive. Second, we find for this potential the exact form: $U_{2}(r) / k_{\mathrm{B}} T=-A_{\mathrm{H}}\left(d_{0} / r\right)^{2} \exp \left(-r / \xi^{*}\right)-B_{\mathrm{H}}\left(d_{0} / r\right)^{4} \exp \left(-2 r / \xi^{*}\right)$, with the known universal amplitudes $A_{\mathrm{H}}>0$ and $B_{\mathrm{H}}>0$ (the Hamaker constants). This expression clearly shows that the pair-potential differs from its homologue with no crosslinks only by the two exponential factors $\exp \left(-r / \xi^{*}\right)$ and $\exp \left(-2 r / \xi^{*}\right)$. The main conclusion is that the presence of reticulations reduces substantially the Casimir effect in crosslinked polymer blends.
\end{abstract}

PACS numbers: $61.25 . \mathrm{Hq}, 64.75 .+\mathrm{g}, 82.70 . \mathrm{Gg}$

\section{Introduction}

Interpenetrated polymer networks (IPNs) or crosslinked polymer blends constitute new materials, which have a considerable application potential. Typical examples are crosslinked epoxy adhesives showing a great resistance to acids, bases and many solvents, and exhibiting high glass transition temperatures and thermal resistance, crosslinked mixtures of bacterial and seaweed polysaccharides gellan and agarose [1], novel IPNs made of polypropylene/poly( $n$-butyl acrylate) [2], or polysiloxane interpenetrating networks used as electronic device encapsulants [3].

For certain practical realizations, the IPNs or crosslinked polymer mixtures are in the presence of colloidal particles, in order to reinforce their mechanical properties. But if these particles prefer to attract strongly one polymer of the crosslinked mixture near the spinodal temperature (critical adsorption [4-20]), one assists to their aggregation in the non-preferred phase. This colloidal flocculation is due to a long-ranged induced potential, called critical Casimir potential we want to compute.

Historically, the Casimir effect was discovered for the first time by Hendrick Casimir in 1948 [21], according to which the vacuum quantum fluctuations of a confined electromagnetic field induce an attractive force between

* corresponding author; e-mail: benhamou.mabrouk@gmail.com two parallel uncharged conducting plates. The Casimir effect has received its final confirmation in more recent experiments by Lamoreaux [22] and by Mohideen and Roy [23].

As noted by Fisher and de Gennes [10], the Casimir effect also appears in the context of statistical and condensed matter physics. More precisely, this effect is inherent to those critical systems, such as a fluid near the liquid-gas critical point, a binary liquid (or a polymer mixture) near the demixing temperature, a liquid ${ }^{4} \mathrm{He}$ near the $\lambda$-transition or liquid crystals. For these systems, the critical fluctuations of the order parameter play the role of vacuum quantum fluctuations, and then give rise to long-ranged forces between the confining walls or between immersed colloids, termed critical Casimir forces in literature [24].

From a theoretical point of view, the Casimir effect related to critical systems confined between two parallel walls or in the presence of immersed colloidal particles, has attracted much attention [10, 24-46]. Experimentally, several works have been devoted to the critical systems in the presence of geometrical boundaries (confining walls or immersed colloids) [47-50]. Very recently, one has also investigated the Casimir effect in critical polymer blends or ternary polymer solutions [51-63].

The physical system we consider here is a polymer blend made of two chemically incompatible polymers $A$ and $B$, crosslinked in their one-phase region (high temperature). We assume that, before the chains are linked each to other, the polymer mixture incorporates colloidal 
particles. The system is prepared in such a way that, near the spinodal temperature, the colloids have some preference to be contacted by one polymer, say $A$. Thus, we are in critical adsorption conditions. To simplify, we suppose that the particle size, $d_{0}=2 R$, is much smaller than the mesh size, $\xi^{*}$, defined later. Below some critical temperature depending on the reticulation dose, the mixture has tendency to phase separate. Because of the presence of permanent crosslinks, the elasticity of the network resists to such a separation. As a result, the crosslinked mixture undergoes a microphase separation (MPS), which is characterized by the appearance of microdomains alternatively rich in $A$ and $B$ polymers.

The first theory of MPS was elaborated by Gennes [64], followed by several extended works [65-78]. The de Gennes trick was an analogy between the crosslinked polymer blend and a dielectric medium. The theoretical predictions were tested by Briber and Bauer [79] by small-angle neutron-scattering experiment on the PS-PVME mixture. The latter was crosslinked by the $\gamma$-ray irradiation tool [80].

In this paper, the purpose is the computation of the Casimir pair-potential between beads that strongly attract the connected polymer $A$ near the spinodal temperature. Such a potential originates from the strong critical fluctuations of the composition, which are present close to this temperature. As consequence, the beads located in the $B$-rich side aggregate.

To achieve the determination of the spatial variation of the induced pair-potential, use is made of an extended model taking into account the colloid-polymer interaction. The associated free energy is a functional of the composition fluctuation $\varphi=\Phi_{A}-\Phi_{B}$, where $\Phi_{A}$ and $\Phi_{B}$ are the respective compositions of $A$ and $B$ polymers. With the help of the standard cumulant method [81, 82], we compute the resulting pair-potential, $U_{2}(r)$, as a function of the interparticle distance $r$. We find for this attractive potential an exact form, which decays with distance $r$ according to: $U_{2}(r)=$ $-A_{\mathrm{H}}\left(d_{0} / r\right)^{2} \exp \left(-r / \xi^{*}\right)-B_{\mathrm{H}}\left(d_{0} / r\right)^{4} \exp \left(-2 r / \xi^{*}\right)$, with known universal amplitudes $A_{\mathrm{H}}>0$ and $B_{\mathrm{H}}>0$, and the mesh size $\xi^{*} \sim a n^{1 / 2}$ ( $a$ being the monomer size and $n-$ the number of monomers between consecutive crosslinks). This exact result clearly shows that the pair-potential differs from its homologue with no crosslinks [56] only by the two exponential factors $\exp \left(-r / \xi^{*}\right)$ and $\exp \left(-2 r / \xi^{*}\right)$. This indicates that the presence of crosslinks reduces considerably the Casimir effect, because the critical fluctuations of the order parameter are less important, when they are compared to those relative to uncrosslinked polymer mixtures.

The remaining presentation proceeds as follows. In Sect. 2, we briefly recall the essential of the de Gennes theory of MPS in the absence of colloids. The presentation of the used model and computation of the Casimir potential are the aim of Sect. 3. Some concluding remarks are drawn in the last section.

\section{De Gennes theory}

We consider two incompatible polymers $A$ and $B$, crosslinked in their one-phase region (at high temperature). When the system is cooled down, below some critical temperature, one assists to the appearance of microdomains alternatively rich in $A$ and $B$ polymers. This MPS results from a competition between the usual macrophase separation and the elasticity of the network.

According to de Gennes [64], the (bulk) free energy governing physics is given by

$$
\frac{\mathcal{H}_{\mathrm{b}}[\varphi]}{k_{\mathrm{B}} T}=\int \mathrm{d}^{d} \boldsymbol{r}\left[\frac{t}{2} \varphi^{2}+\kappa(\nabla \varphi)^{2}-\frac{C}{2} \varphi \Delta^{-1} \varphi\right],
$$

with $T$ - the absolute temperature and $k_{\mathrm{B}}$ - the Boltzmann constant. The temperature parameter

$$
t=a^{-2} \frac{1}{2}\left(\chi_{\mathrm{c}}-\chi\right)
$$

is proportional to the distance from the critical point, where $\chi$ is the standard Flory interaction parameter, and $\chi_{\mathrm{c}}=N / 2$ its critical value, when the mixture is uncrosslinked. Here, $N$ is the common polymerization degree of $A$ and $B$ chains. Notice that the parameter $\chi$ is essentially the inverse of temperature. The gradient term describes the interfacial energy between $A$-rich and $B$-rich phases, with the coefficient $\kappa=1 / 9$. There, $a$ is the monomer size and $d$ - the space dimensionality. The field or order parameter $\varphi(\boldsymbol{r})$ is directly proportional to the local fluctuation of composition $\rho(\boldsymbol{r})=\Phi(\boldsymbol{r})-\Phi_{\mathrm{c}}$, where $\Phi$ is the composition of one component of the mixture and $\Phi_{\mathrm{c}}=1 / 2$ its critical value. We recall that the first two terms in relation (1) constitute the expansion of the usual Flory-Huggins free energy [83, 84] around the critical composition, and the third one describes the gel elasticity. The elastic contribution in this relation was first introduced by de Gennes by analogy with the polarization of a dielectric medium. The internal rigidity constant $C$ was related to the average number $n$ of monomers per strand by [64]

$$
C \sim n^{-2} a^{-4} \text {. }
$$

By strand, we mean the section of chains between consecutive crosslinks. In formula (1), $\Delta^{-1}$ represents the inverse of the Laplacian operator, and acts as

$$
\Delta_{r}^{-1} \varphi(\boldsymbol{r})=\int \mathrm{d}^{d} \boldsymbol{r}^{\prime} \mathcal{G}\left(\boldsymbol{r}-\boldsymbol{r}^{\prime}\right) \varphi\left(\boldsymbol{r}^{\prime}\right),
$$

where the propagator $\mathcal{G}\left(\boldsymbol{r}-\boldsymbol{r}^{\prime}\right)$ is solution to the differential equation

$$
\Delta_{r} \mathcal{G}\left(\boldsymbol{r}-\boldsymbol{r}^{\prime}\right)=\delta_{d}\left(\boldsymbol{r}-\boldsymbol{r}^{\prime}\right) .
$$

In reciprocal space, the free energy (1) becomes

$$
\frac{\mathcal{H}_{\mathrm{b}}\left[\varphi_{q}\right]}{k_{\mathrm{B}} T}=\frac{1}{2} \sum_{\boldsymbol{q}} S^{-1}(\boldsymbol{q})\left|\varphi_{q}\right|^{2},
$$

with

$$
S(q)=\frac{1}{2 \kappa q^{2}+t+\frac{C}{q^{2}}}
$$

the structure factor [64]. It differs from the usual one $(C=0)$ by the extra term $C / q^{2}$ that describes the presence of crosslinks. The above structure factor exhibits a 
maximum for

$$
q^{*}=\left(\frac{C}{2 \kappa}\right)^{1 / 4} .
$$

The quantity $\xi^{*}=q^{*-1} \sim a \sqrt{n}$ is interpreted as the size of microdomains (mesh size). The above formula indicates that, contrarily to macrophase separation, the MPS takes place at $q \neq 0$. The maximum value of the structure factor, $S\left(q^{*}\right)$, diverges at the spinodal for

$$
t_{\mathrm{s}}=-2 \sqrt{2 \kappa C} \text {. }
$$

In the following section, we will be interested in the computation of the Casimir potential between colloidal particles trapped in a critical crosslinked polymer blend.

\section{Casimir force}

\subsection{The field model}

Consider a low-density assembly of spherical colloids of the same diameter $d_{0}=2 R$, which are dispersed in a crosslinked polymer mixture. For the sake of simplicity, we assume that the particle diameter $d_{0}$ is much smaller than the mesh size $\xi^{*} \sim a \sqrt{n}$, where $n$ is the number of monomers per strand. We suppose that near the spinodal temperature, the particles prefer to be contacted by the polymer $A$. As a result, the beads located in the non-preferred phase inside microdomains aggregate. This colloidal aggregation is then caused by a long-ranged critical Casimir potential. The aim of this work is precisely the computation of such a potential upon the interparticle distance. Since the critical fluctuations occur inside microdomains, the potential has important values only for distances smaller than the mesh size.

To compute the expected potential, the starting point is the free energy that describes the crosslinked polymer blend with immersed colloids

$$
\begin{aligned}
& \frac{\mathcal{H}[\varphi]}{k_{\mathrm{B}} T}=\frac{\mathcal{H}_{\mathrm{b}}[\varphi]}{k_{\mathrm{B}} T}+\frac{g_{0}}{2} \sum_{i=1}^{M} \int \mathrm{d} S_{i} \varphi_{i}^{2} \\
& -\mu_{0} \sum_{i=1}^{M} \int \mathrm{d} S_{i} \varphi_{i},
\end{aligned}
$$

with $\mathcal{H}_{\mathrm{b}}[\varphi]$ - the de Gennes free energy, relation (1). In the above equality, $\mathrm{d} S_{i}$ is the elementary measure on the surface of colloid $i$, and $M$ - the total number of colloids. There, $\varphi_{i}$ is the value of field $\varphi$ on the surface of colloid $i$, $g_{0}$ - the coupling constant measuring the interaction strength between surfaces of colloids and polymer chains, and $\mu_{0}$ the difference of surface chemical potentials of components $A$ and $B$. We note that the surface terms in Eq. (8) are those usually encountered in surface critical phenomena $[16,17,85]$.

A considerable simplification occurs when the particles are point-like. In this case, the surface field $\varphi_{i}$ becomes $\varphi\left(\boldsymbol{r}_{i}\right)$, where $\boldsymbol{r}_{i}$ is the position vector of the center of mass of colloid $i$. With these considerations, the new free energy reads

$$
\frac{\mathcal{H}[\varphi]}{k_{\mathrm{B}} T}=\frac{\mathcal{H}_{\mathrm{b}}[\varphi]}{k_{\mathrm{B}} T}+\frac{g}{2} \sum_{i=1}^{M} \varphi^{2}\left(\boldsymbol{r}_{i}\right)-\mu \sum_{i=1}^{M} \varphi\left(\boldsymbol{r}_{i}\right) .
$$

Therefore, the parameters of the problem are $(t, g, \mu)$, with the rescaled surface parameters $g=g_{0} s_{d} R^{d-1}$ and $\mu=\mu_{0} s_{d} R^{d-1}$, where $s_{d} R^{d-1}$ is the area of a sphere (colloid) of radius $R=d_{0} / 2$ embedded in a $d$-dimensional space, with $s_{d}=2 \pi^{(d / 2)} / \Gamma(d / 2)$. The critical adsorption emerges in the limit $\mu \rightarrow \infty$, at fixed coupling constant $g>0$ [85]. This limit defines the so-called normal transition [85].

\subsection{Effective pair-potential}

To compute the Casimir force, we start with the partition function of $M$ colloids, of fixed positions $\left(\boldsymbol{r}_{1}, \ldots, \boldsymbol{r}_{\mathrm{M}}\right)$ in space,

$$
\mathcal{Z}\left(\boldsymbol{r}_{1}, \ldots, \boldsymbol{r}_{\mathrm{M}}\right)=\int \mathcal{D} \varphi \mathrm{e}^{-\mathcal{H}[\varphi] / k_{\mathrm{B}} T},
$$

with $\mathcal{H}[\varphi]$ - the Hamiltonian defined in Eq. (9). The basic object is the effective free energy of colloids, $\mathcal{F}_{\mathrm{M}}\left(\boldsymbol{r}_{1}, \ldots, \boldsymbol{r}_{\mathrm{M}}\right)$, defined through the standard relation

$$
\mathcal{F}_{\mathrm{M}}\left(\boldsymbol{r}_{1}, \ldots, \boldsymbol{r}_{\mathrm{M}}\right)=-k_{\mathrm{B}} T \ln \mathcal{Z}\left(\boldsymbol{r}_{1}, \ldots, \boldsymbol{r}_{\mathrm{M}}\right) \text {. }
$$

But the quantity of interest is rather the difference

$$
\begin{aligned}
& \Delta \mathcal{F}_{\mathrm{M}}\left(\boldsymbol{r}_{1}, \ldots, \boldsymbol{r}_{\mathrm{M}}\right) \\
& \quad=\mathcal{F}_{\mathrm{M}}\left(\boldsymbol{r}_{1}, \ldots, \boldsymbol{r}_{\mathrm{M}}\right)-\sum_{i=1}^{M} \mathcal{F}_{1}\left(\boldsymbol{r}_{i}\right) .
\end{aligned}
$$

Here, $\mathcal{F}_{1}\left(\boldsymbol{r}_{i}\right)$ is the free energy to confine one particle in the crosslinked mixture. In fact, the quantity $\Delta \mathcal{F}_{\mathrm{M}}\left(\boldsymbol{r}_{1}, \ldots, \boldsymbol{r}_{\mathrm{M}}\right)$ is nothing else but the total mutual interaction energy

$$
\begin{gathered}
\Delta \mathcal{F}_{\mathrm{M}}\left(\boldsymbol{r}_{1}, \ldots, \boldsymbol{r}_{\mathrm{M}}\right)=\frac{1}{2} \sum_{i, j} U_{2}\left(\boldsymbol{r}_{i}, \boldsymbol{r}_{j}\right) \\
+\frac{1}{3 !} \sum_{i, j, k} U_{3}\left(\boldsymbol{r}_{i}, \boldsymbol{r}_{j}, \boldsymbol{r}_{k}\right)+\ldots,
\end{gathered}
$$

with $U_{p}(p=2,3, \ldots)$ - the $p$-body interaction potential between particles. Here, we restrict ourselves to two-body interactions, only.

Introduce the bulk mean value, $\langle X\rangle_{\mathrm{b}}$, of a functional $X[\varphi]$ of the field configuration $\varphi$ :

$$
\langle X\rangle_{\mathrm{b}}=\mathcal{Z}_{\mathrm{b}}^{-1} \int \mathcal{D} \varphi X[\varphi] \mathrm{e}^{-\mathcal{H}_{\mathrm{b}}[\varphi] / k_{\mathrm{B}} T},
$$

where

$$
\mathcal{Z}_{\mathrm{b}}=\int \mathcal{D} \varphi \mathrm{e}^{-\mathcal{H}_{\mathrm{b}}[\varphi] / k_{\mathrm{B}} T}
$$

is the partition function of the crosslinked polymer blend free from particles. With the help of this definition, the partition function rewrites

$$
\mathcal{Z}\left(\boldsymbol{r}_{1}, \ldots, \boldsymbol{r}_{\mathrm{M}}\right)=\mathcal{Z}_{\mathrm{b}} \times\left\langle\mathrm{e}^{\mathcal{O}\left(\boldsymbol{r}_{1}, \ldots, \boldsymbol{r}_{\mathrm{M}}\right)}\right\rangle_{\mathrm{b}},
$$

with the notation

$$
\mathcal{O}\left(\boldsymbol{r}_{1}, \ldots, \boldsymbol{r}_{\mathrm{M}}\right)=-\frac{g}{2} \sum_{i=1}^{M} \varphi^{2}\left(\boldsymbol{r}_{i}\right)+\mu \sum_{i=1}^{M} \varphi\left(\boldsymbol{r}_{i}\right) .
$$

Using the cumulant techniques [82, 83], based on the approximative formula 


$$
\begin{aligned}
& \langle\exp (X)\rangle_{\mathrm{b}} \\
& \quad=\exp \left(\langle X\rangle_{\mathrm{b}}+\frac{1}{2}\left[\left\langle X^{2}\right\rangle_{\mathrm{b}}-\langle X\rangle_{\mathrm{b}}^{2}\right]+\ldots\right),
\end{aligned}
$$

we show that

$$
\begin{aligned}
& \frac{\Delta \mathcal{F}_{\mathrm{M}}\left(\boldsymbol{r}_{1}, \ldots, \boldsymbol{r}_{\mathrm{M}}\right)}{k_{\mathrm{B}} T}=-\frac{\mu^{2}}{2} \sum_{i, j=1}^{M} G_{1}\left(\boldsymbol{r}_{i}-\boldsymbol{r}_{j}\right) \\
& -\frac{g^{2}}{8} \sum_{i, j=1}^{M} G_{2}\left(\boldsymbol{r}_{i}-\boldsymbol{r}_{j}\right)
\end{aligned}
$$

with

$$
\begin{aligned}
& G_{1}\left(\boldsymbol{r}_{i}-\boldsymbol{r}_{j}\right)=\left\langle\varphi\left(\boldsymbol{r}_{i}\right) \varphi\left(\boldsymbol{r}_{j}\right)\right\rangle_{\mathrm{b}}-\left\langle\varphi\left(\boldsymbol{r}_{i}\right)\right\rangle_{\mathrm{b}}\left\langle\varphi\left(\boldsymbol{r}_{j}\right)\right\rangle_{\mathrm{b}} \\
&=\int \frac{\mathrm{d}^{d} \boldsymbol{q}}{(2 \pi)^{d}} \frac{\mathrm{e}^{\mathrm{i} \boldsymbol{q} \cdot\left(\boldsymbol{r}_{i}-\boldsymbol{r}_{j}\right)}}{2 \kappa q^{2}+t+\frac{C}{q^{2}}}
\end{aligned}
$$

and

$$
\begin{aligned}
G_{2} & \left(\boldsymbol{r}_{i}-\boldsymbol{r}_{j}\right) \\
& =\left\langle\varphi^{2}\left(\boldsymbol{r}_{i}\right) \varphi^{2}\left(\boldsymbol{r}_{j}\right)\right\rangle_{\mathrm{b}}-\left\langle\varphi^{2}\left(\boldsymbol{r}_{i}\right)\right\rangle_{\mathrm{b}}\left\langle\varphi^{2}\left(\boldsymbol{r}_{j}\right)\right\rangle_{\mathrm{b}} .
\end{aligned}
$$

Therefore, comparing Eqs. (13) to (17) yields

$$
\frac{U_{2}\left(\boldsymbol{r}-\boldsymbol{r}^{\prime}\right)}{k_{\mathrm{B}} T}=-\mu^{2} G_{1}\left(\boldsymbol{r}-\boldsymbol{r}^{\prime}\right)-\frac{g^{2}}{4} G_{2}\left(\boldsymbol{r}-\boldsymbol{r}^{\prime}\right) .
$$

This fundamental relation expresses that the Casimir potential is a linear combination of the bulk correlation functions constructed with the field $\varphi$ and the composite operator $\varphi^{2}$, respectively. The same relation explains why the Casimir effect emerges from the fluctuations of composition. The negative sign in the above equality means that the effective potential is attractive. Notice that the two correlation functions are positive definite. This attraction is then responsible for the colloidal aggregation in the non-preferred phase. Also, we note that the dependence of the expected pair-potential on the polymer-colloid interaction is entirely contained in the squared surface parameters $\mu^{2}$ and $\mathrm{g}^{2}$. Using scale invariance property of the correlation functions, it was recently found that these parameters are given by [60]:

$$
g=\frac{1024 \pi^{4}}{243} R^{2} N, \quad \mu=\frac{32 \pi^{2}}{9 \sqrt{3}} R \sqrt{N} .
$$

Formula (20) then suggests that the computation of the pair-potential necessitates the knowledge of the correlation functions $G_{1}(\boldsymbol{r})$ and $G_{2}(\boldsymbol{r})$. But the latter can be related to the former by application of the Wick theorem usually encountered in field theory [81, 82], that is

$$
G_{2}(\boldsymbol{r})=2\left[G_{1}(\boldsymbol{r})\right]^{2} .
$$

We show, in Appendix, that the two-point correlation $G_{1}(r)$, at the spinodal temperature, is exactly given by

$$
G(r)=\frac{1}{16 \pi^{2} \kappa} \frac{1}{\xi^{* 2}}\left[\frac{2 \xi^{*}}{r} K_{1}\left(\frac{r}{\xi^{*}}\right)-K_{0}\left(\frac{r}{\xi^{*}}\right)\right],
$$

with $\xi^{*} \sim a \sqrt{n}$ - the mesh size, and $K_{\nu}(z)$ - the modified Bessel function [86]. Since, the essential of the phenomenon occurs in the critical fluctuations domain of size $\xi^{*}$. At these small scales, the above correlation reduces to

$$
G(r)=\frac{1}{8 \pi^{2} \kappa} \frac{1}{r^{2}} \mathrm{e}^{-r / \xi^{*}}, \quad r<\xi^{*} .
$$

We have used the behaviors $K_{0}(z) \sim-\ln z$ and $K_{1}(z) \sim$ $\mathrm{e}^{-z} / z$ of the modified Bessel functions, for $z \rightarrow 0$ [86].

With these considerations, the pair-potential writes

$$
\begin{aligned}
& \frac{U_{1}(r)}{k_{\mathrm{B}} T}=-A_{\mathrm{H}}\left(\frac{d_{0}}{r}\right)^{2} \mathrm{e}^{-r / \xi^{*}}-B_{\mathrm{H}}\left(\frac{d_{0}}{r}\right)^{4} \mathrm{e}^{-2 r / \xi^{*}}, \\
& r<\xi^{*}
\end{aligned}
$$

with the universal amplitudes

$$
A_{\mathrm{H}}=\frac{32 \pi^{2}}{27} N, \quad B_{\mathrm{H}}=\frac{512 \pi^{4}}{729} N^{2} .
$$

Let comment about the expression (22) of the pair-potential.

Firstly, notice that the appearance of $r^{-2}$ and $r^{-4}$ -powers factors in formula $(22)$ that multiply the exponential tails $\exp \left(-r / \xi^{*}\right)$ and $\exp \left(-2 r / \xi^{*}\right)$, is related to the small-distance behaviors of the correlation functions $G_{1,2}(r): G_{1}(r) \sim r^{-2 x_{\varphi}}$ and $G_{2}(r) \sim r^{-2 x_{\varphi^{2}}}\left(r \ll \xi^{*}\right)$. Indeed, at small distances compared to the mesh size $\xi^{*}$, the reticulations cannot be felt. Here, $x_{\varphi}=\beta_{t} / \nu_{t}$ and $x_{\varphi^{2}}=d-1 / \nu_{t}$ are the respective scaling dimensions of fields $\varphi$ and $\varphi^{2}$, where $\beta_{t}$ and $\nu_{t}$ are the standard Ising critical exponents $[81,82]$ describing the behaviors of composition fluctuation and thermal correlation length. In mean-field theory (of our interest), $x_{\varphi}=1$ and $x_{\varphi^{2}}=2$. Therefore, the negative power laws are completely determined knowing the values of the above scaling dimensions.

Secondly, this potential may be compared to the usual Landé potential that governs the attraction between a pair of (dipolar) molecules, which decays rather as $r^{-6}$. This means that the calculated Casimir force is much greater than the traditional dispersed force, at any distance.

Thirdly, expression (22) reveals that the pair-potential is a van der Waals interaction that is screened by the presence of permanent crosslinks. Thus, the amplitudes $A_{\mathrm{H}}$ and $B_{\mathrm{H}}$ play the role of the Hamaker constants.

Fourthly, the dependence on the shape of colloids manifests itself through their diameter $d_{0}=2 R$ appearing in relation $(22)$. Then, shapely particles attract each other strongly in comparison with point-like ones. This can be understood noting that the bigger colloids are surrounded by an important quantity of the preferred polymer near the spinodal temperature.

Fifthly, the dependence on the reticulation dose $n^{-1}$ of the pair-potential is entirely contained in the mesh size $\xi^{*} \sim a n^{1 / 2}$. This fact tells us that the Casimir effect is more pronounced only for weakly crosslinked polymer mixtures.

Sixthly, the Casimir potential of crosslinked polymer blends is less important than that of their homologue at $C=0$ (absence of reticulations). This is natural, since the presence of reticulations reduces considerably the critical fluctuations of the order parameter.

Finally, in the limit $C \rightarrow 0\left(\xi^{*} \rightarrow \infty\right)$, we recover the usual result [56]. 
In Fig. 1, we report the variations of the reduced Casimir potential, $U_{2}(r) / k_{\mathrm{B}} T$, upon the renormalized interparticle distance $r / D$, for a crosslinked polymer mixture (solid curve) and an uncrosslinked one (dashed curve). Here the notation $D=d_{0} \sqrt{N}$ means a renormalized particle size. For the first curve, the chosen mesh size is such as $D / \xi^{*}=10$.

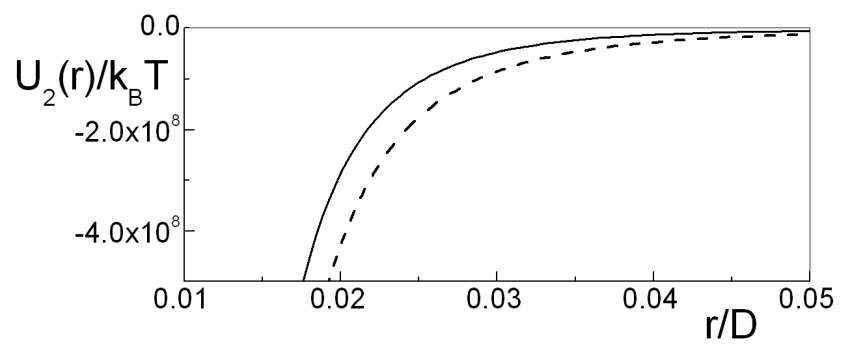

Fig. 1. Reduced pair-potential, $U_{2}(r) / k_{\mathrm{B}} T$, versus the renormalized interparticle distance $r / D$, for a crosslinked polymer mixture (solid curve) and an uncrosslinked one (dashed curve). For the first curve, the chosen mesh size is such as $D / \xi^{*}=10$.

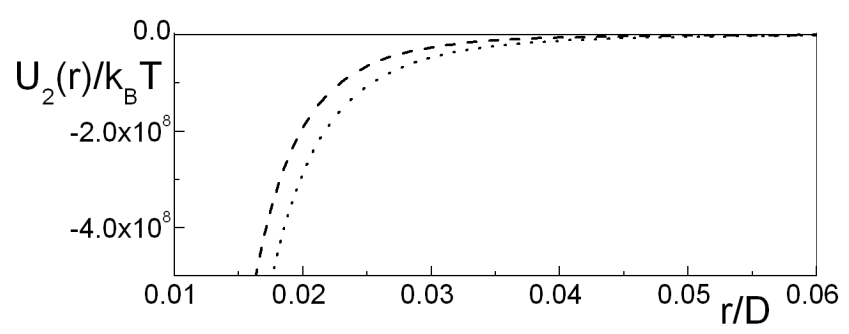

Fig. 2. Reduced pair-potential, $U_{2}(r) / k_{\mathrm{B}} T$, versus the renormalized interparticle distance $r / D$, for two crosslinked polymer mixtures of respective ratios $D / \xi_{1}^{*}=10$ (dotted curve) and $D / \xi_{2}^{*}=20$ (dashed curve).

In Fig. 2, we superpose the variations of the reduced Casimir potential, $U_{2}(r) / k_{\mathrm{B}} T$, upon the renormalized interparticle distance $r / D$, for two crosslinked polymer mixtures of respective ratios $D / \xi_{1}^{*}=10$ (dashed curve) and $D / \xi_{2}^{*}=20$ (solid curve).

The shapes of various curves reflect the discussions made above.

\section{Conclusions}

We recall that the purpose of the present work was the investigation of the Casimir effect in crosslinked polymer blends. This effect originates from the critical fluctuations of the order parameter within these systems. As consequence, particles immersed in the non-preferred phase aggregate. This flocculation is provoked by an effective Casimir pair-potential we have computed.

We note that we have not discussed the variations of the Casimir potential, for distances beyond the mesh size $\xi^{*}$. The reason is that, at these scales, the Casimir effect cease to exist due to the absence of critical fluctuations.

We point out that the presence of the exponential factors in expression (22) is not surprising. Indeed, their appearance can be interpreted as a finite size effect due to the restricted domain of critical fluctuations.

We emphasize that the pair-potential expression we derived can be extended to two particles of different diameters $d_{1}$ and $d_{2}$. For this case, we state that the new expression would be similar to that relative to a monodisperse colloidal system, making the following change of the diameter $d_{0}: d_{0} \rightarrow \sqrt{d_{1} d_{2}}$.

Finally, open problems such as solvent and temperature effects are under consideration.

\section{Acknowledgments}

We are much indebted to Professors M. Daoud for fruitful discussions, and to S. Dietrich, E. Eisenriegler and M. Krech for useful correspondences. We are much indebted to our referee for his important remarks and useful suggestions.

\section{Appendix}

The aim of this appendix is to demonstrate formula (21) defining the two-point correlation $G_{1}(r)$. The starting point is its Fourier representation

$$
G_{1}(r)=\int \frac{\mathrm{d}^{d} \boldsymbol{q}}{(2 \pi)^{d}} \frac{\mathrm{e}^{\mathrm{i} \boldsymbol{q} \cdot \boldsymbol{r}}}{2 \kappa q^{2}+t+\frac{C}{q^{2}}} .
$$

It is convenient to write the propagator in reciprocal space as

$$
\frac{1}{t+2 \kappa q^{2}+\frac{C}{q^{2}}}=\frac{A}{q^{2}+\alpha}+\frac{B}{q^{2}+\beta},
$$

with the notations

$$
\begin{aligned}
& A=\frac{1}{2 \kappa} \frac{\alpha}{\alpha-\beta}, \quad B=\frac{1}{2 \kappa} \frac{\beta}{\beta-\alpha}, \\
& \alpha=\frac{t+\sqrt{t^{2}-8 \kappa C}}{4 \kappa}, \quad \beta=\frac{t-\sqrt{t^{2}-8 \kappa C}}{4 \kappa} .
\end{aligned}
$$

With these considerations, the two-point correlation rewrites

$$
G_{1}(r)=A \int \frac{\mathrm{d}^{d} \boldsymbol{q}}{(2 \pi)^{d}} \frac{\mathrm{e}^{\mathrm{i} \boldsymbol{q} \cdot \boldsymbol{r}}}{q^{2}+\alpha}+B \int \frac{\mathrm{d}^{d} \boldsymbol{q}}{(2 \pi)^{d}} \frac{\mathrm{e}^{\mathrm{i} \boldsymbol{q} \cdot \boldsymbol{r}}}{q^{2}+\beta} .
$$

Thus, the function $G_{1}(r)$ is a linear combination of the Fourier transforms of the usual propagators with squared masses $\alpha$ and $\beta$. We recall simply that [56]:

$$
\begin{aligned}
& \int \frac{\mathrm{d}^{d} \boldsymbol{q}}{(2 \pi)^{d}} \frac{\mathrm{e}^{\mathrm{i} \boldsymbol{q} \cdot \boldsymbol{r}}}{q^{2}+\alpha}=(2 \pi)^{-d / 2}\left(\frac{r^{2}}{\alpha}\right)^{\nu / 2} K_{\nu}(r \sqrt{\alpha}), \\
& \int \frac{\mathrm{d}^{d} \boldsymbol{q}}{(2 \pi)^{d}} \frac{\mathrm{e}^{\mathrm{i} \boldsymbol{q} \cdot \boldsymbol{r}}}{q^{2}+\beta}=(2 \pi)^{-d / 2}\left(\frac{r^{2}}{\beta}\right)^{\nu / 2} K_{\nu}(r \sqrt{\beta}),
\end{aligned}
$$

with the order $\nu=(2-d) / 2$. 
For crosslinked polymer blends, the criticality emerges in the $d \rightarrow 4$ limit. We find that, in this limit, the two-point correlation is given by

$$
\begin{aligned}
& G_{1}(r)=\frac{1}{8 \pi^{2} \kappa} \frac{1}{\alpha-\beta} \\
& \quad \times\left[\frac{\alpha^{3 / 2}}{r} K_{1}(r \sqrt{\alpha})-\frac{\beta^{3 / 2}}{r} K_{1}(r \sqrt{\beta})\right] .
\end{aligned}
$$

On the other hand, at the spinodal temperature, that is at $t=t_{\mathrm{s}}=-2 \sqrt{2 \kappa C}$, we have $\alpha=\beta=\xi^{*-2}$, and then, expression (A.6) reduces to

$$
G_{1}(r)=\left.\frac{1}{8 \pi^{2} \kappa} \frac{1}{r} \frac{\mathrm{d}}{\mathrm{d} \alpha}\left[\alpha^{3 / 2} K_{1}(r \sqrt{\alpha})\right]\right|_{\alpha=\xi^{*-2}} .
$$

Now, using certain mathematical properties of the modified Bessel function [86], we show the desired relation (21).

This ends the proof of the expected formula.

\section{References}

[1] E. Amici, A.H. Clark, V. Normand, N.B. Johnson, Biomacromolecules 1, 721 (2000).

[2] C. Zhao, M. Xu, W. Zhu, X. Luo, Polymer 39, 275 (1998).

[3] G.J. Gibbons, D. Holland, J. Sol-Gel Sci. Technol. 8, 599 (1997).

[4] D. Beysens, S. Leibler, J. Phys. Lett. (Paris) 43, L-133 (1982).

[5] C. Franck, S.E. Schnatterly, Phys. Rev. Lett. 48, 763 (1982).

[6] M. Schlossman, X.-L. Wu, C. Franck, Phys. Rev. B 31, 1478 (1985).

[7] J.A. Dixon, M. Schlossman, X.-L. Wu, C. Franck, Phys. Rev. B 31, 1509 (1985).

[8] An extensive list of recent experiments using some new techniques can be found in: A. Hanke, M. Krech, F. Schlesener, S. Dietrich, Phys. Rev. E 60, 5163 (1999).

[9] S. Blümel, G.H. Findenegg, Phys. Rev. Lett. 54, 447 (1985).

[10] M.E. Fisher, P.-G. de Gennes, C.R. Acad. Sci. (Paris) Sér. B 287, 207 (1978); P.-G. de Gennes, C.R. Acad. Sci. (Paris) II 292, 701 (1981).

[11] E. Brézin, S. Leibler, Phys. Rev. B 27, 594 (1983).

[12] S. Leibler, Ph.D. Thesis, Paris XI University, 1984.

[13] J. Rudnik, D. Jasnow, Phys. Rev. Lett. 48, 1059 (1982).

[14] S. Leibler, L. Peliti, J. Phys. C 15, L-403 (1982).

[15] L. Peliti, S. Leibler, J. Phys. C 16, L-2635 (1983).

[16] H.W. Diehl, in: Phase Transitions and Critical Phenomena, Eds. C. Domb, J.L. Lebowitz, Vol. 10, Academic Press, London 1986.

[17] S. Dietrich, in: Phase Transitions and Critical Phenomena, Eds. C. Domb, J.L. Lebowitz, Vol. 12, Academic Press, London 1988.
[18] Critical adsorption on curved objects, such as single spherical and rod-like colloidal particles, has been investigated in: A. Hanke, S. Dietrich, Phys. Rev. E 59, 5081 (1999); A. Hanke, Phys. Rev. Lett. 84, 2180 (2000).

[19] H. Zhao, A. Penninckx-Sans, L.-T. Lee, D. Beysens, G. Jannink, Phys. Rev. Lett. 75, 1977 (1995).

[20] S. Blümel, G.H. Findenegg, Phys. Rev. Lett. 54, 447 (1985).

[21] H.B.G. Casimir, Proc. Kon. Ned. Akad. Wetenschap B 51, 793 (1948).

[22] S.K. Lamoreaux, Phys. Rev. Lett. 78, 5 (1997).

[23] U. Mohideen, A. Roy, Phys. Rev. Lett. 81, 4549 (1998).

[24] M. Krech, The Casimir Effect in Critical Systems, World Sci., Singapore 1994.

[25] N.M. Barber, in: Phase Transitions and Critical Phenomena, Eds. C. Domb, M.S. Green, Vol. 8, Academic Press, New York 1983.

[26] E. Brézin, J. Phys. (Paris) 43, 15 (1982).

[27] V. Privman, in: Finite Size Scaling and Numerical Simulation of Statistical Systems, Ed. V. Privman, World Sci., Singapore 1990.

[28] M. Krech, S. Dietrich, Phys. Rev. A 46, 1886 (1992).

[29] M. Krech, Phys. Rev. E 56, 1642 (1997).

[30] H.W.J. Blöte, J.L. Cardy, M.P. Nightingale, Phys. Rev. Lett. 56, 742 (1986).

[31] I. Affleck, Phys. Rev. Lett. 56, 746 (1986).

[32] J.L. Cardy, Nucl. Phys. B 275, 200 (1986).

[33] T.W. Burkhardt, T. Xue, Phys. Rev. Lett. 66, 895 (1991).

[34] T.W. Burkhardt, T. Xue, Nucl. Phys. B 345, 653 (1991).

[35] T.W. Burkhardt, E. Eisenriegler, Nucl. Phys. B 424 [FS], 487 (1994).

[36] K. Symanzik, Nucl. Phys. B 190 [FS], 1 (1981).

[37] M. Krech, S. Dietrich, Phys. Rev. Lett. 66, 345 (1991).

[38] M. Krech, S. Dietrich, Phys. Rev. Lett. 67, 1055 (1991).

[39] M. Krech, S. Dietrich, Phys. Rev. A 46, 1922 (1992).

[40] E. Eisenriegler, M. Stapper, Phys. Rev. B 50, 10009 (1994).

[41] T.W. Burkhardt, E. Eisenriegler, Phys. Rev. Lett. 74, 3189 (1995).

[42] E. Eisenriegler, U. Ritschel, Phys. Rev. B 51, 13717 (1995).

[43] K.K. Mon, Phys. Rev. Lett. 54, 2671 (1985).

[44] M. Krech, D.P. Landau, Phys. Rev. E 53, 4414 (1996).

[45] R.R. Netz, Phys. Rev. Lett. 76, 3646 (1996).

[46] F. Schlesener, A. Hanke, S. Dietrich, J. Stat. Phys. 110, 981 (2003).

[47] D. Beysens, D. Estève, Phys. Rev. Lett. 54, 2123 (1985).

[48] V. Gurfein, D. Beysens, F. Perrot, Phys. Rev. A 40, 2543 (1989) 
[49] M.L. Broide, Y. Garrabos, D. Beysens, Phys. Rev. E 47, 3768 (1993).

[50] T. Narayanan, A. Kumar, E.S.R. Gopal, D. Beysens, P. Guenoun, G. Zalczer, Phys. Rev. E 48, 1989 (1993).

[51] A. Saout Elhak, R. Cherrabi, M. Benhamou, M. Daoud, J. Chem. Phys. 111, 8174 (1999).

[52] R. Cherrabi, A. Saout Elhak, M. Benhamou, M. Daoud, Phys. Rev. E 62, 6795 (2000).

[53] R. Cherrabi, Ph.D. Thesis, Hassan II-Mohammedia University, 1998.

[54] A. Saout Elhak, Ph.D. Thesis, Hassan II-Mohammedia University, 2000.

[55] E.-K. Hachem, M. Benhamou, M. Daoud, J. Chem. Phys. 116, 8168 (2002).

[56] H. Ridouane, E.-K. Hachem, M. Benhamou, J. Chem. Phys. 118, 10780 (2003).

[57] H. Ridouane, E.-K. Hachem, M. Benhamou, Condens. Matter Phys. 7, 63 (2004).

[58] H. Ridouane, Ph.D. Thesis, Hassan II-Mohammedia University, 2004.

[59] E.-K. Hachem, Ph.D. Thesis, Hassan II-Mohammedia University, 2004.

[60] M. Benhamou, H. Ridouane, A. Derouiche, M. Rahmoune, J. Chem. Phys. 122, 244913 (2005).

[61] M. Benhamou, M. El Yaznasni, H. Ridouane, E.K. Hachem, Braz. J. Phys. 36, 1 (2006).

[62] M. El Yaznasni, H. Ridouane, E.-K. Hachem, M. Benhamou, N. Chafi, M. Benelmostafa, Phys. Chem. News, in press.

[63] M. El Yaznasni, H. Ridouane, E.-K. Hachem, M. Benhamou, J. Maghr. Phys., in press.

[64] P.-G. de Gennes, J. Phys. Lett. (Paris) 40, 69 (1979).

[65] A. Bettachy, A. Derouiche, M. Benhamou, M. Daoud, J. Phys. II (Paris) 1, 153 (1991).

[66] A. Derouiche, A. Bettachy, M. Benhamou, M. Daoud, Macromolecules 25, 7188 (1992).

[67] T.A. Vilgis, M. Benmouna, M. Daoud, M. Benhamou, A. Bettachy, A. Derouiche, Polym. Network Blends 3, 59 (1993).

[68] M. Benhamou, Int. J. Mod. Phys. A 8, 2581 (1993).
[69] M. Benmouna, T.A. Vilgis, M. Daoud, M. Benhamou, Macromolecules 27, 1172 (1994).

[70] M. Benmouna, T.A. Vilgis, M. Benhamou, A. Babaoui, M. Daoud, Macromol., Theory Simul. 3, 557 (1994)

[71] A. Bettachy, A. Derouiche, M. Benhamou, M. Benmouna, T.A. Vilgis, M. Daoud, Macromol., Theory Simul. 4, 67 (1995).

[72] M. Benhamou, J. Chem. Phys. 102, 5854 (1995).

[73] D.J. Read, M.G. Brereton, T.C.B. McLeish, J. Phys. II (Paris) 5, 1679 (1995)

[74] A. Bettachy, Ph.D. Thesis, Hassan II-Mohammedia University, 1995.

[75] A. Derouiche, Ph.D. Thesis, Hassan II-Mohammedia University, 1995.

[76] M. Riva, V.G. Benza, J. Phys. II (Paris) 7, 285 (1997).

[77] M. Benhamou, A. Derouiche, A. Bettachy, J. Chem. Phys. 106, 2513 (1997).

[78] A. Derouiche, M. Benhamou, A. Bettachy, Eur. Phys. J. E 13, 353 (2005).

[79] R.M. Briber, B.J. Bauer, Macromolecules 21, 3296 (1988).

[80] M. Dole, The Radiation Chemistry of Macromolecules, Vol. I and II, Academic Press, New York 1972.

[81] C. Itzykson, J.-M. Drouffe, Statistical Field Theory: 1 and 2, Cambridge University Press, Cambridge 1989.

[82] J. Zinn-Justin, Quantum Field Theory and Critical Phenomena, Clarendon Press, Oxford 1989.

[83] P.J. Flory, Principles of Polymer Chemistry, Cornell University Press, Ithaca 1953.

[84] P.-G. de Gennes, Scaling Concepts in Polymer Physics, Cornell University Press, Ithaca 1979.

[85] K. Binder, in: Phase Transitions and Critical Phenomena, Eds. C. Domb, J.L. Lebowitz, Vol. 8, Academic Press, London 1983.

[86] I.S. Gradshteyn, I.M. Ryzik, Table of Integrals, Series and Products, Academic Press, New York 1980. 